\title{
DAS ICH DER INSTINKTE IN HUSSERLS MANUSKRIPTEN ÜBER DIE LEBENSWELT
}

TAKUYA NAKAMURA

\begin{abstract}
The I of instincts in Husserl's manuscripts on the Lifeworld

In this paper, I attempt to elucidate the phenomenological approach to the problem of primal beginning (Uranfang) in the late Husserl with reference to his analysis of the ego of instincts. The first part deals with the instinct referring to the work of Nam-In Lee. It makes clear that the concept of instinct in Husserl is not the object of psychological analysis, but the authentic phenomenological concept. The second part starts with trying to interpret thoroughly the text no. 43 in Husserl's Lebenswelt (Husserliana Vol. XXXIX). Thereby, the antero-posterior relationship of the ego and the streaming, or "I think" and "I am" respectively, is explained. The third part explains what the primal beginning of transcendental subjectivity is. Drawing on the method of construction, Husserl discovers the primal beginning of transcendental subjectivity as the intertwining of instinct and affection.
\end{abstract}

Dieses Verzeitlichen ist selbst strömendes; das Strömen ist immerzu im Voraus. Aber auch das Ich ist im Voraus, es ist als waches Ich (transzendental-phänomenologisch wach) immerfort Bewusstseins-Ich. ${ }^{1}$

Diese Aussage Edmund Husserls, die die paradoxe und zirkelhafte Seinsweise des Ichs behandelt, wurde bei Forschern durch die Zitierung von Klaus Held aus einem damals nicht veröffentlichten Manuskript Husserls, dem sogenannten

1 Husserl Edmund, Zur phänomenologischen Reduktion. Texte aus dem Nachlass (1926-1935), Dordrecht, Boston, London, Kluwer Academic Publishers, Husserliana. Bd. XXXIV, 2002, S. 181. Siehe das Zitat dieser Stelle aus dem damals unpublizierten Manuskript durch Held. Held Klaus, Lebendige Gegenwart. Die Frage nach der Seinsweise des transzendentalen Ich bei Edmund Husserl, entwickelt am Leitfaden der Zeitproblematik, Den Haag, Martinus Nijhoff, Phaenomenologica 23, 1966, S. $110 f$.

https://doi.org/10.14712/24646504.2019.9

(C) 2019 The Author. This is an open-access article distributed under the terms of the Creative Commons Attribution License (http://creativecommons.org/licenses/by/4.0). 
C-Manuskript, bekannt. Er verwies auf diese Stelle sehr eindringlich in seiner klassischen Monographie Lebendige Gegenwart. Diese Stelle hat schon viele verschiedene Interpretationen in der Husserl-Forschung verursacht.

Die genannte Aussage Husserls über das Strömen und das Ich zeigt das Rätsel, auf das Husserl in der letzten Periode seines Lebens gestoßen ist, nämlich das Rätsel, das in den tiefsten Schichten der transzendentalen Subjektivität entsteht. Es ist die rätselhafte Seinsweise des transzendentalen Ichs, welche die Phänomenologie auf ihre Grenze stoßen lässt. Aus einer anderen Perspektive erhellt ein Studium von Nam-In Lees Edmund Husserls Phänomenologie der Instinkte ein und dasselbe Rätsel. Dieses Werk betrachtet das Rätsel als das Phänomen der Instinkte, das ebenfalls in der tiefsten Schicht der transzendentalen Subjektivität entsteht. Es ist das Problem des Anfangs als genetischer Ursprung.

Seit der Publikation des Buchs von Lee wurde das Phänomen des Instinktes als ein Hauptproblem in Husserls Phänomenologie bekannt. Husserl behandelt es als Thema seiner Analysen der 1930er Jahre. Dies bestätigt die Wichtigkeit dieses Phänomens beim späten Husserl.

Die Aufgabe dieses Aufsatzes ist, den Horizont des Problems des Ichs und der Instinkte, den Lee als erster erhellt hat, an Husserls Text orientiert zu erweitern. Zwar enthalten Husserls Grenzprobleme der Phänomenologie. Analyse des Instinktes. Analysen des Unbewusstseins und der Instinkte. Metaphysik. Späte Ethik. Texte aus dem Nachlass (1908-1937) die relevanten Texte. Aber laut dem Herausgeber dieses Bandes enthalten auch die Späten Texte über Zeitkonstitution (1929-1934), Die C-Manuskripte und Die Lebenswelt. Auslegungen der vorgegebenen Welt und ihrer Konstitution. Texte aus dem Nachlass (1916-1937) wichtige Texte, die sich auf diese Problematik beziehen. ${ }^{2}$

Wenn auch die Manuskripte durch den Herausgeber als Texte über Instinkte aufgenommen wurden, haben sie unvermeidlich fragmentarischen Charakter, weil diese Materialien aus Forschungsmanuskripten stammen. Der vorliegende Aufsatz behandelt nicht die Gesamtheit dieser Manuskripte, die bisher als Gedanken des späten Husserl angesehen werden, sondern orientiert sich ausschließlich an Manuskript Nr. 43 im Husserliana Bd. XXXIX. Dieses Manuskript enthält die sehr feinteilige Analyse der Instinkte und Affektionen im Zusammenhang mit dem Ich. Durch die Auslegung dieses Manuskripts will ich einen wichtigen Aspekt des husserlschen Denkens als Resultat aus den ungeheuren Mengen seiner Gedanken und seiner Analyse der Instinktproblematik in diesem Manuskript herausheben. Die

2 Sowa Rochus „Einleitung“, in Husserl E., Grenzprobleme der Phänomenologie. Analysen des Unbewusstseins und der Instinkte. Metaphysik. Späte Ethik. Texte aus dem Nachlass (1908-1937), Heidelberg, New York, London, Springer, Husserliana Bd. XLII, 2014, S. XLII, Anm. 2. 
Aufgabe des vorliegenden Aufsatzes ist demnach, durch Husserls Analyse des Instinktes die Affektion und in ihrem Zusammenhang die Vor- und Nachbeziehung von Ich und Strömen aufzuklären.

Der vorliegende Aufsatz behandelt Folgendes: Erstens zeigt er, dass im Ausgang von Lees ${ }^{3}$ Studie der Instinkt nicht das Thema der psychologischen Analyse, sondern der unverzichtbar wichtige Begriff ist, der nach einer Phänomenologie als transzendentaler Philosophie verlangt. Das heißt zugleich die Veränderung und Erweiterung der kantisch transzendentalen Philosophie durch Husserl. Zweitens wird erklärt, wie sich die Frage nach dem Anfang der Subjektivität beim späten Husserl stellt. Das Manuskript Nr. 43 stellt die Frage nach der Vor- und Nachbeziehung von Ich und Strömen, wie das anfängliche Zitat gezeigt hat. Dies stellt sich als zirkelhaft-schwierige Problematik des Anfangs der transzendentalen Subjektivität dar. Drittens wird erhellt, was es ist, worauf Husserl letztlich gemäß seiner Fragestellung und seines Ansatzes beim Anfang der transzendentalen Subjektivität gestoßen ist. Dabei erwäge ich, was die Methode der Konstruktion ist, die Husserl eingeführt hat, um die Frage nach dem Anfang zu stellen und seine Analyse nachzuvollziehen. Danach wird verdeutlicht, dass das Ich des durch diese Konstruktion gewonnenen Uranfangs das Ich der Instinkte ist. Letztlich wird im Hinblick auf die vorausgehende Betrachtung problematisiert, ob eine solche Sache wie das Ich im Uranfang als Verflechtung von Affektion und Instinkt das Ziel ist, das den späten Husserl beruhigen kann und in dem sein letztes Denken aufhören dürfte. Ich will zeigen, dass eine solche Verflechtung nicht der Endpunkt für die phänomenologische Analyse ist, sondern vielmehr ihr Ausgangspunkt, welcher der Phänomenologie die weitere Frage aufgibt.

\section{Der Instinkt als transzendentaler Begriff}

In der transzendentalen Phänomenologie Husserls, insbesondere in seiner genetischen Phänomenologie, wird nun der Instinkt von Forschern als unerlässlicher Begriff angesehen. Aber wenn man die transzendentale Philosophie nur im kantisch Sinne fasst, ist es nicht selbstverständlich, dass der Instinkt ein transzendentaler Begriff ist. Darüber hinaus „wäre die Phänomenologie der Instinkte als eine transzendentale Theorie ein Unding “4 . Denn von der transzendentalen Philosophie im kantisch Sinne aus wird der Instinkt gar nicht als transzendentaler

3 Lee Nam-In, Edmund Husserls Phänomenologie der Instinkte, Dordrecht, Boston, London, Kluwer Academic Publishers, Phaenomenologica 128, 1993.

4 Ibid., S. 3. 
Begriff angesehen, sondern er ist nur ein psychologischer Begriff und gehört nicht zum transzendentalen, sondern zum empirischen Bewusstsein. Also würde der Instinkt, der zum empirischen Bewusstsein gehört, nicht in die transzendentale Philosophie eingeordnet.

Bei Kant ist zwar der Gegenbegriff zu transzendental empirisch, aber bei Husserl ist, wie Eugen Fink herausgestellt hat, der Gegenbegriff zu transzendental nicht empirisch, sondern „mundan“5. In der transzendentalen Philosophie Husserls sind die beiden Begriffe transzendental und empirisch voneinander nicht klar unterschieden. Dies ist dadurch offenbar, dass Husserl in seiner Krisis-Abhandlung den Bereich des Erfahrungsbegriffs erweitert. Im Hinblick auf den Aufweis Finks sagt Lee: „Das nach Kant als empirisch bestimmte Bewußtsein kann sich nach Husserl je nach der Einstellung nicht nur als mundanes, sondern auch als transzendentales Bewußtsein zeigen"6. In diesem Sinne fasst Husserl den Instinkt in seiner genetischen Phänomenologie, welche die Genesis des transzendentalen Bewusstseins zum Thema hat, als transzendentalen Begriff, und die transzendentale Funktion des Instinkts als den fundamentalen Grundteil auf, welcher der transzendentalen Phänomenologie zugrunde liegt. Er erkennt die unerlässliche Bedeutung des Instinkts für das ganze System der transzendentalen Phänomenologie an. ${ }^{7}$ Wenn auch der Instinkt als das Transzendentale vom Standpunkt der kantisch transzendentalen Philosophie aus undenkbar wäre, weist dies darauf hin, „daß zwischen der Transzendentalphilosophie Kants und der transzendentalen Phänomenologie Husserls trotz der Ähnlichkeit und Verwandtschaft ein Unterschied, [...] ein unüberbrückbarer Unterschied, besteht "8. Wenn ausschließlich die kantisch transzendentale Philosophie im eigentlichen Sinne anerkannt würde und andere Typen

5 Fink Eugen, „Die Phänomenologie Edmund Husserls in der gegenwärtigen Kritik“, in Ders., Studien zur Phänomenologie 1930-1939, Den Haag, Martinus Nijhoff, Phaenomenologica 21, 1966, S. 147.

6 Lee N.-I., Edmund Husserl Phänomenologie der Instinkte op. cit., S. 220.

7 Die Einleitung des Herausgebers von Husserliana Bd. XV. Zur Phänomenologie der Intersubjektivität. Texte aus dem Nachlass. Dritter Teil: 1929-1935 enthält „Disposition zu ,SYSTEM DER PHÄNOMENOLOGISCHEN PHILOSOPHIE' von Edmund Husserl“ (S. XXXVI-XL). Dort findet man, dass die „Phänomenologie der Urintentionalität (Phänomenologie der ,Instinkte')“ an der entscheidenden Stelle dieser Disposition steht.

8 Lee N.-I., Edmund Husserl Phänomenologie der Instinkte op. cit., S. 4. Darüber hinaus zeigt Lee die Diskrepanz von Kant und Husserl, genauer gesagt, die Zustimmung Husserls zu Kant in der genetischen Phänomenologie und die Diskrepanz von Husserl und Kant in der genetischen Phänomenologie im Hinblick auf den Aufweis Finks (s.o.) auf (ibid., S. 221). Dann zeigt er, dass Husserl Leibniz näherkommt. „Die transzendentale Phänomenologie als eine universale Philosophie im echten Sinne, welche alle möglichen Positionen im Spannungsfeld zwischen transzendentalem Intellektualismus und Voluntarismus umspannt, nennt Husserl in der Spätphilosophie in Anlehnung an Leibniz die transzendentale Monadologie“ (ibid., S. 246). 
der transzendentalen Philosophie verneint würden, liefe dies darauf hinaus, die transzendentale Philosophie zu eng zu begreifen. ${ }^{9}$

Es ist schwer zu sagen, dass das Phänomen der Instinkte sowohl im $\mathrm{Zu}-$ sammenhang mit der Geschichte der Philosophie als auch mit der Husserl-Forschung nicht treffend und ihrem Wert entsprechend behandelt wurde. Vor der umfangreichen Studie Lees über die Problematik der Instinkte bei Husserl gab es doch sehr wenig Forschung über diesen Begriff. Wenn der Instinkt behandelt wird, ist seine Analyse unzutreffend ${ }^{10}$ oder berücksichtigt nicht die eng auf den Instinkt bezogenen Begriffe, ${ }^{11}$ wie Lee dargestellt hat. Lee hat die wichtige Position der Phänomenologie der Instinkte in der ganzen transzendentalen Phänomenologie orientiert an der „Disposition zu SYSTEM DER PHÄNOMENOLOGISCHEN PHILOSOPHIE von Edmund Husserl" (Hua XV xxxvi -xl) aufgewiesen. Erst danach wird das Phänomen der Instinkte als ein wichtiges Thema in der Husserl-Forschung anerkannt. Und dazu wird durch das Erscheinen von Hua Bd. XLII Grenzprobleme der Phänomenologie, wo Manuskripte bezüglich des Instinktes als zweiter Teil der Ausgabe versammelt sind, die Forschung zu diesem Thema weiter entwickelt. ${ }^{12}$ Das bis hierhin Gesagte gibt einen Überblick über die bisherige Lage der Forschung zum Instinkt in der Phänomenologie Husserls. Im Folgenden will ich mich mit der Analyse des Ichs und des Instinkts bei Husserl selbst beschäftigen.

9 Vgl. Heinämaa Sara, Hartimo Mirja und Miettinen Timo (Hrsg.), Phenomenology and the Transcendental, London and New York, Routledge, Routledge Research in Phenomenology, 2014 und Gardner Sebastian und Grist Matthew (Hrsg.), The Transcendental Turn, Oxford, Oxford University Press, 2015.

10 Die Monographie von Alwin Diemer, die die Phänomenologie Husserls in der früheren Periode der Husserl-Forschung systematisch zu beschreiben versucht, ordnet die Phänomenologie der Instinkte nicht in die genetische, sondern in die statische Phänomenologie ein. Und deshalb verliert sie den eigentlichen Wert im System der Phänomenologie überhaupt aus dem Blick (Lee N.-I., Edmund Husserl Phänomenologie der Instinkte op. cit., S. 9). Diemer Alwin, Edmund Husserl. Versuch einer systematischen Darstellung seiner Phänomenologie, 2. verb. Auflage, Meisenheim am Glan, Verlag Anton Hain, 1965, S. 98 ff.

11 In seiner Phänomenologie der Assoziation, welche die sich auf Affektion und Instinkt tief beziehende Assoziation als Hauptthema behandelt, analysiert Holenstein "den untrennbaren Zusammenhang der Instinktphänomene und der Assoziationsphänomene" nicht eingehend (Lee, N.-I., Edmund Husserl Phänomenologie der Instinkte op. cit., S. 10). Holenstein Elmar, Die Phänomenologie der Assoziation. Zur Struktur und Funktion eines Grundprinzips der passiven Genesis bei E. Husserl, Den Haag, Martinus Nijhoff, Phaenomenologica 44, S. 37, Anm. 15.

12 Z. B. sei an die folgenden Forschungen erinnert, die den Instinkt und den Trieb als Thema behandeln. Pugliese Alice, „Triebsphäre und Urkindheit des Ich“, in Husserl Studies 25, 2009, S. 141-157. Brower Matt, "Husserl's Theory of Instincts as a Theory of Affection", in The Journal of the British Society for Phenomenology, 45, 2014, S. 133-147. 


\section{Die Frage nach dem Anfang der Subjektivität. Das zirkelhafte Phänomen des Strömens und des Ichs}

Husserliana Bd. XXXIX Lebenswelt Nr. 43 „Das Problem des Anfangs der (primordialen) Subjektivität. Anfangende Affektion als instinktive. Methode der Rekonstruktion (zur Methode transzendental-ästhetischer Auslegung)"13 wurde laut dem Herausgeber im Januar 1931 geschrieben. ${ }^{14}$ Im Folgenden versuche ich, dieses Manuskript intensiv auszulegen, welches nicht allzu diskrepant und fragmentarisch ist und überdies mit dem Thema des Instinkts eng zusammenhängt und obwohl es ein Manuskript des späten Husserl ist - eine relativ hohe Kohärenz aufweist. Dieser Text steht zwischen den beiden folgenden Texten: Husserliana Bd. XXXIV Nr. 10 „Zur lebendigen Gegenwart. Passive Zeitigung des Erlebnisstroms gegenüber der Verzeitlichung der Akte. Vorzeitigung und eigentliche Zeitigung "15, aus dem ich die erste zitierte Stelle in diesem Aufsatz entnommen habe und der im Sommer 1930 entstand, und dem Zusatz dazu aus dem Jahre 1932. ${ }^{16}$ Die Periode des Verfassens von Manuskript Nr. 43 fällt genau in die Zwischenzeit. In diesem Sinne gehören beide Forschungsmanuskripte zur gleichen Periode im Denken Husserls der 1930er Jahre. Die Aufgabenstellung Husserls in Manuskript Nr. 43 ist deutlich. Dort wird die Verwandtschaft mit dem anfänglichen Zitat gefunden.

Abstrahieren wir in den individuellen (und dabei in strömender totaler Konstitution einheitlich genommenen) Weltaspekten von den „Anderen“, reduzieren wir also auf das Primordiale, so kommen wir auf das primordiale Phänomen „Welt“ im primordialen Ego; wir kommen <auf> die subjektive Weltzeitlichkeit mit dem eigenen subjektiv konstituierten Leib und menschlichen Subjekt und auf das Problem des Anfangs; und wenn wir die Konstitution thematisch machen, <kommen wir $>$ zum Problem des Anfangs des wachen transzendentalen Ego und seiner transzendentalen Zeitlichkeit des Strömens und im Strömen „innere“ Zeit und zeitliches Sein von „Daten“ Konstituierens. ${ }^{17}$

Die zweite Hälfte ist weniger eindeutig und muss deshalb sorgfältig gedeutet werden. Jedenfalls ist das Thema dieses Manuskripts der Anfang „des wachen tran-

13 Husserl E., Die Lebenswelt. Auslegungen der vorgegebenen Welt und ihrer Konstitution. Texte aus dem Nachhass (1916-1937), The Netherlands, Springer, Husserlina Band XXXIX, S. 466-482.

14 Ibid., S. 466, Anm. 1.

15 Husserl E., Zur phänomenologischen Reduktion. Texte aus dem Nachlass (1926-1935), Dordrecht, Boston, London, Kluwer Academic Publishers, Husserliana. Bd. XXXIV, 2002, S. 179-184.

16 Ibid., S. 184.

17 Husserl E., Die Lebenswelt. Auslegungen der vorgegebenen Welt und ihrer Konstitution, op. cit., S. $466 f$. 
szendentalen Ego und seiner transzendentalen Zeitlichkeit des Strömens“. Und das Strömen des „wachen transzendentalen Ego“ wird konkreter als „im Strömen ,innere' Zeit und zeitliches Sein von ,Daten' Konstituierens" angesehen. Der Zusammenhang dieser drei Begriffe - „wach[es] transzendentale[s] Ego“, das „Strömen“ und die „innere“ Zeit sowie zeitliches Sein von „Daten“ Konstituieren - ist nichts anderes als das Problem dieses Manuskripts. Aber das Strömen enthält zweierlei, nämlich das transzendentale Ego und das Konstituieren, als seine Bestände. In diesem Sinne bilden diese beiden Begriffe die Konkretion des Strömens. Das transzendentale Ego als das Konstituieren konstituiert „,innere“ Zeit“, die dem Ego eigen ist, und jede und alle außer dem Ego selbst nämlich als, „zeitliches Sein von ,Daten“'. Dabei wird dem Ego und allem außer dem Ego durch das Konstituieren des transzendentalen Ego eine gewisse Zeitstelle gegeben, das heißt, es wird „verzeitlicht". Diese Verzeitlichung durch das Konstituieren des transzendentalen Ego bildet das Strömen. Dieser ganze Verlauf ist nichts anderes als die Konkretion des Strömens. Aber das Konstituieren des transzendentalen Ego verzeitlicht sich selbst dadurch, dass das Konstituieren wiederum auch das Konstituieren selbst konstituiert. Demnach lässt das transzendentale Ego im Strömen Strom entstehen und zugleich sich selbst entgleiten, nämlich verzeitlichen. An dieser Stelle stellt Husserl eine Frage: Wie fängt dieses Strömen, nämlich „die transzendentale Zeitlichkeit“ an? Dies ist das Problem der „transzendentalen Zeitlichkeit“. Hier soll darauf aufmerksam gemacht werden, dass Husserl sehr gezielt an dieser Stelle das transzendentale Ego „waches transzendentales Ego“ nennt. Wach bedeutet, dass das Ego schon durch die Affektion vom Nicht-Ichlichen aus angefangen hat, ein Interesse für das Affizierende zu haben und sich ihm zuzuwenden. Dieses wache transzendentale Ego ist also schon irgendwie durch das Nicht-Ichliche affiziert worden und hat angefangen, sich ihm aktiv zuzuwenden.

Wenn es sich so verhält, dann muss das Affizierende, vor dem Konstituierenden und vor dem Verzeitlichenden, dem wachen transzendentalen Ego vorausgehen. Dieses das wache transzendentale Ego Affizierende befindet sich nicht in der Phase der Konstitution im normalen und üblichen Sinne, nämlich der Konstitution der Einheit von weltlicher Transzendenz. Deshalb muss Husserl die Frage nach der Phase des Affizierenden vertiefen: „Betrachten wir jetzt aber die Ur-Affektionen, die der vorobjektiven Stufe, näher" ${ }^{18}$. Hier spricht Husserl explizit davon, dass er die Frage nach der der Objektivierung vorausgehenden Phase der „Ur-Affektion, die der vorobjektiven Stufen“, stellt. Deswegen ist die „Ur-Affektion“, die hier problematisiert wird, nichts anderes als die aller Objektivierung,

18 Ibid., S. 469. 
die Verzeitlichung als die niedrigste Stufe der Konstitution enthält, vorausgehende Sache. Sie ist ganz anders als die Affektion, die bei der Konstitution der Einheit von der weltlichen Transzendenz im üblichen Sinne problematisiert wird. Durch die Vertiefung der Frage nach der Phase der aller Objektivierung vorausgehenden Sache tritt das Problem der Vor- und Nachbeziehung des Strömens und des Ego als der Vor- und Nachbeziehung von „Ich bin“ und „Ich denke“ in den Vordergrund.

Sage ich apodiktisch „Ich bin“, indem ich „denke“, dass ich bin, und indem ich irgendein „Ich denke“ vollziehe, so liegt darin doch auch apodiktisch, dass das „Ich denke“ Affektion voraussetzt und dass ich sein muss, um affiziert werden zu können und wenn von mir etwas soll erfasst werden können. ${ }^{19}$

Zunächst sieht es so aus, als ermögliche das „Ich denke“ das „Ich bin“. Aber dieses „Ich denke“ bedeutet genauer „,ich denke, dass ich bin“. „[D]ass das ,Ich denke‘ Affektion voraussetzt“" klingt so, als ermögliche das „Ich bin“ das „Ich denke“ dadurch, dass das „Ich bin“ mich affiziert. Um affiziert zu werden oder etwas aktiv zu erfassen, muss ich (zuerst) sein. Wenn das so ist, scheint die Vor- und Nachbeziehung von "Ich bin“ und „Ich denke“ eine unauflösbar zirkelhafte Beziehung zu sein. Es folgen die Sätze, die den Stellenwert dieses Problems deutlich machen:

Aber ist darin „Werden“ nicht erdichtet, als ob eine Zeit schon vorausgezeitigt wäre?
Zeitigung setzt das urphänomenale Strömen voraus, dass, wenn es verläuft, eben
schon Zeit zeitigt, die also mit ihm schon da ist. Aber wie soll ein unendliches Strö-
men konstituiert sein ohne „Abgehobenheit“ und Affektion? Es handelt sich doch
um nichts Minderes als um den Anfang der Zeitkonstitution, und zwar den Anfang
einer „subjektiven“Zeit in einer anfangenden Zeitigung, die, obschon anfangend, doch
nicht besagen kann: in einer Zeitlichkeit, die schon ist, - also im gewöhnlichen Sinne
anfangend. ${ }^{20}$

An dieser Stelle wird „Ich denke“ als „Zeitigung“, genauer gesprochen, als „das urphänomenale Strömen“ als Voraussetzung thematisiert. Und es scheint so zu sein, dass „das urphänomenale Strömen“ gerade die Urvoraussetzung in dem Sinne ist, dass es die Zeitigung ermöglicht. Aber danach kommt das Problem des Anfangs des urphänomenalen Strömens als Urvoraussetzung zum Vorschein. Das das urphänomenale Strömen Affizierende, nämlich das "Ich bin“ im letzten Zitat,

19 Ibid., S. 470.

20 Idem. 
wird zur Voraussetzung des „unendlichen Strömens“. Daraus wird deutlich, dass das diese zirkelhafte Aporie betreffende Problem des Anfangs das letzte Problem, das auf „den Anfang einer ,subjektiven' Zeit in einer angefangenen Zeitigung " hier antworten soll.

Von den bisherigen Zitaten aus wird das Problem deutlich. Das Problem der Vor- und Nachbeziehung des Ichs und des Strömens verändert sich zu der Vorund Nachbeziehung des wachen transzendentalen Ego und der Uraffektion der vorobjektiven Stufe, und weiter zum „Ich denke“ und „Ich bin“. Diese Vor- und Nachbeziehungen sind die zirkelhafte Aporie des Anfangs. Darüber hinaus ist die Dimension, in der diese Aporie problematisiert wird, nichts anderes als der „Anfang einer, subjektiven' Zeit in einer anfangenden Zeitigung". Er ist nicht der Anfang im üblichen Sinne innerhalb des Rahmens von Zeitigen und Gezeitigtem. Nach der Klarstellung des Problems arbeitet Husserl die radikale Methode aus, um „den Anfang einer ,subjektiven' Zeit in einer anfangenden Zeitigung" eingehend zu behandeln.

\section{Instinkt-Affektion als Uranfang}

Um das Problem des „Anfang[s] der ,subjektiven 'Zeit“ aufzuklären, muss sich Husserl die Methode für die Zusammenfassung der Struktur dieses Problems radikal überlegen. Und dabei muss man darauf aufmerksam sein, dass der Begriff der Konstruktion als bedeutender Begriff in den Vordergrund tritt, um in den Anfang einzudringen.

Wirklich für mich als zeitlich seiend (oder, was dasselbe, als seiend) konstituiert ist, was aus meiner konstituierenden Aktivität her konstituiert worden ist, und eventuell vermittels apperzeptiver Übertragung seinen Zeitsinn hat. Konstituierende Aktivität setzt aber passive Zeitkonstitution voraus, und wir werden zurückgeführt auf eine solche passive Konstitution, die vorzeitlich vorseiend schon die Zeitlichkeit in sich trägt. Aber wesensmäßig können wir, nachdem wir Seiendes in wirklicher Zeitlichkeit schon haben, reflektierend zurückgehen und aufgrund der Wiedererinnerung und in ihrer Wiederholung nachträglich zeitliche Konstitution vollziehen, also das zeitliche Sein als Vergangenheit der nachherigen Gegenwart konstruieren. Das aber in Evidenz als eine „vergessene“, aktuell nicht vollzogen gewesene, aber wirkliche Vergangenheit. Das betrifft ja die gesamte anonyme Bewusstseinssphäre, die sogar zum größten Teil erst durch die phänomenologische Arbeit zu einer „expliziten“ Zeitigung kommt, während es ihr im Leben an wirklich für das Ich < seiendem> zeitlichem Sein fehlte. Es heißt dann aber mit Evidenz: Es war auch für mich gewesen und ist weiter für mich mit da, 
wo ich es nicht „beachte“, es war implizite schon für mich seiend. Und „seiend“ heißt nun für mich überhaupt $<$ das $>$, was von explizit konstituiertem Sein <aus> rückgewendet zur Zeitigung gebracht werden kann, in meinem freien „Ich kann“ und „kann immer wieder".21

Zwar wird die phänomenologische Analyse durch die Reflexion charakterisiert, aber hier wird die phänomenologische Analyse, die durchaus die phänomenologische Reflexion genannt wird, im Hinblick auf den konkreten Entstehungsprozess in Ordnung gebracht. Zuerst beginnt die phänomenologische Analyse mit dem Besitz des „Seiende[n] in wirklicher Zeitlichkeit“ als das, „was aus meiner konstituierenden Aktivität her konstituiert worden ist". Durch den Vollzug der Reflexion darauf wird man dann , auf eine solche passive Konstitution, die vorzeitlich vorseiend schon die Zeitlichkeit in sich trägt", zurückgeführt. In diesem Sinne geht die passive Konstitution der aktiven Konstitution voraus. Aber in Wirklichkeit hat man zuerst „Seiendes in wirklicher Zeitlichkeit", und erst dann kann man auf den Grund dieses „Seienden in wirklicher Zeitlichkeit" reflektierend zurückgehen. In diesem Sinne gehen also die aktive Konstitution und ihre Resultate der passiven Konstitution voraus.

Die zeitliche Konstitution, die erst durch den Vollzug dieses Rückgangs ermöglicht wird, nennt Husserl überraschenderweise in diesem Zusammenhang nicht „Konstituieren“, sondern „Konstruieren“: „Aber wesensmäßig können wir [...] reflektierend zurückgehen und aufgrund der Wiedererinnerung und in ihrer Wiederholung nachträglich zeitliche Konstitution vollziehen, also das zeitliche Sein als Vergangenheit der nachherigen Gegenwart konstruieren". Und dazu wird diese ganze Reihe der zeitlichen Konstitution "die phänomenologische Arbeit“ genannt. Durch diese phänomenologische Arbeit wird „die gesamte anonyme Bewusstseinssphäre“ für das Ego (aber doch zugleich „[war es] implizite schon für mich seiend“) als etwas, ,was von explizit konstituiertem Sein <aus $>$ rückgewendet zur Zeitigung gebracht werden kann", nämlich als ein quasizeitliches Seiendes, konstruiert. Aber die Konstruktion als die phänomenologische Arbeit bezieht sich noch auf die Gegenwart in ihrem weiteren Sinne. Denn die Konstruktion ist hier der Übergang von der expliziten Bewusstseinssphäre als der Gegenwart im engeren Sinne dessen, worauf ich aufmerksam bin, zu der impliziten Bewusstseinssphäre, also dem, worauf ich nicht aufmerksam bin, die trotz alledem die Gegenwart im weiteren Sinne ist, durch die Konstruktion des zeitlichen Seins als Vergangenheit der nachherigen Gegenwart. Diese Konstruktion des zeitlichen Seins als Vergangenheit der nachherigen Gegenwart bedeutet nur den Übergang

21 Ibid., S. 470f. 
von der Explizität zur Implizität in der zeitlichen Modalität der Gegenwart. Aber das Problem des Anfangs der subjektiven Zeit, das hier dahingehend thematisiert werden soll, ob es explizit oder implizit konstituiert werde, muss sich überhaupt nicht auf zeitliche Konstitution, sondern auf die gleichsam nicht-zeitliche Phase vor dieser zeitlichen Konstitution beziehen. Trotzdem versucht Husserl, diese Konstruktion auf das Problem des Anfangs anzuwenden.

Inwiefern können wir nun (und wenn wir wirklich können, müssen wir auch) diese Methode auch anwenden zur Zeitigung unseres Anfangs - der doch nicht wirklich ein Anfang im natürlichen Sinne ist -, ja, in gewisser Weise sogar zur Zeitigung des Ich des Anfangs und „vor“ dem Anfang, wo von einem „vor“ im eigentlichen Sinn gar keine Rede sein kann?22

Die die phänomenologische Analyse ermöglichende phänomenologische Arbeit macht die anonyme Bewusstseinssphäre explizit durch die Zurückwendung von explizit konstituiertem Sein auf die Zeitigung. Anders gesagt gibt sie dem Ich ein zeitliches Sein durch die Zeitigung. Aber das vorliegende Problem des Anfangs der subjektiven Zeit ist das Problem des Anfangs, der vor der Genesis des explizit konstituierten Seins steht. Warum versucht Husserl die Methode der Konstruktion, die üblicherweise auf den Anfang im natürlichen Sinne angewandt wird, auf die „Zeitigung des Ich des Anfangs und ,vor' dem Anfang, wo von einem ,vor 'im eigentlichen Sinn gar keine Rede sein kann“, anzuwenden? Der Anfang vor dem zeitlichen Anfang als Anfang im natürlichen Sinne ist nicht natürlich, und zwar als nicht-zeitliches Sein. Zwischen dem Anfang vor der Zeit einerseits und dem zeitlichen Anfang andererseits gibt es eine Kluft. Im Hinblick hierauf kann man die Methode der Konstruktion, die allein von der Explizität aus in die Implizität innerhalb der zeitlichen Ebene zurückleitet, auf den Anfang vor der Zeit grundsätzlich nicht anwenden.

Aber die Methode dieser Konstruktion ist eigentlich der Rückgang von der Gegenwart im engeren Sinne auf die Gegenwart im weiteren Sinne. In diesem Sinne hat sie eine gewisse Ähnlichkeit als Methode des Rückgangs mit dem Rückgang vom zeitlichen Sein zum vorzeitlichen Vorsein, wodurch das Problem des Anfangs vor der Zeit erhellt werden kann. Es bleibt also noch die Möglichkeit, die Konstruktion auf die Zeitigung des Ichs des Anfangs und vor dem Anfang anzuwenden. Dabei kann man unter der Konstruktion die Methode der rückschreitenden Konstitution des Vorseins ohne zeitliches Sein von dem zeitlich konstituierten Sein aus verstehen. Husserl sagt:

22 Ibid., S. 471. 
Und so versuchen wir auch, weiter zu sehen und Evidenz dafür herzustellen, dass rückgreifende evidente Zeitkonstitution von einer Strecke oder Stelle schon konstituierter Zeitlichkeit (konstituierten Seins) <aus> auch in dieses erste Strömen und seinen Anfang und sein vorausgesetztes Ich der Affektion Zeitigung und Sein hineinbringen muss und somit auch das Vorseiende als seiend in Anspruch nehmen $<$ muss > - in einem entsprechend geänderten Sinn gegenüber dem natürlichen (der Vorgegebenheit). ${ }^{23}$

Die Konstruktion, als Stellen der Frage nach dem Anfang, geht, wie mit dem Ausdruck „rückgreifend“ eindringlich ausgedrückt wird, von „schon konstituierter Konstitution" sowie dem Übergang von der expliziten Bewusstseinssphäre zur impliziten Bewusstseinssphäre aus, auf das vorzeitliche Vorsein, nämlich das „erste Strömen und seinen Anfang und sein vorausgesetztes Ich der Affektion". Ähnlich wie „das Ich“, dem an „<seiendem> zeitlichem Sein fehlte“, zu einer „expliziten“ Zeitigung kommt, wird man durch die Konstruktion dazu geführt, Zeitigung und Sein ins Vorseiende hineinzubringen. Durch die Konstruktion wird schließlich auf das Vorseiende, das „erste Strömen und seinen Anfang und sein vorausgesetztes Ich der Affektion" zurückgegriffen. Was ist denn der Anfang, auf den schließlich zurückgegriffen wird, im letzten Sinne?

Das affizierte Ich hat in der Anfangsaffektion (oder den mehreren Affektionen) noch nicht ein Ich-Leben und ein Etwas, woraufhin es lebt. - Es erwacht zum Leben, lebt aber noch nicht, sondern erst, wenn die strömende passive und die aktive Konstitution ihr Wirken getan hat, ein Wirken, das selbst Wirken des konkreten Ich ist. Genauer: Ich ist immer schon Ich von Vermögen, Ich von Kinästhesen, aber auch immer schon Ich, das ein Nicht-Ichliches, ein Hyletisches hat. ${ }^{24}$

Diese „Anfangsaffektion“, die durch die erweiterte Anwendung der Konstruktion auf das Vorsein endlich geborgen wird, ist der Anfang vor dem Anfang der Zeitlichkeit. Sie - in diesem Sinne und auf dieser Ebene - ist gleichsam der Uranfang. Aber auch auf der Ebene dieses Uranfangs scheint die Vor- und Nachbeziehung noch ein Problem zu sein, weil auch auf dieser Ebene noch das "Ich-Leben“ und „Etwas, woraufhin es lebt" unterschieden sind. Und Dieses ermögliche Jenes. Aber dieses Verhältnis ist nicht so einfach als zeitliche Vor- und Nachbeziehung auf der schon gezeigten Ebene zu verstehen. Der Uranfang, der erst nach dem Hineinbringen von Zeitigung und Sein in ihn durch die Konstruktion thematisiert werden kann, ist eigentlich und prinzipiell überhaupt nicht zeitlich. Wie kann

23 Ibid., S. 471.

24 Ibid., S. 472. 
man die Vor- und Nachbeziehung anders denn als zeitliche Beziehung in dieses Nicht-Zeitliche hineinbringen? Treffend erfasst Husserl diese Schwierigkeit.

Die Assoziation fungiert nicht. Das , anfangende Ich“ hat keinen Vergangenheitsuntergrund. Es ist ohne jede Zeitigung. Und das Erste der Affektion, das erste Datum, ist nicht apperzipierbar als irgendein Seiendes, in der Art der Vorgegebenheit der Dinge, die ein natürlich erwachendes Ich affizieren aufgrund der Empfindungsdaten, die ihre Auffassungen wecken etc., womit zugleich der Zusammenhang des Lebens über die Schlaflücke sich synthetisch zusammenschließt. ${ }^{25}$

Im Uranfang fungiert die Assoziation, die die Grundfunktion des Bewusstseins in der passiven Synthesis ist, nicht. Der Grund dafür ist, dass es dem anfangenden Ich vor der Zeitigung des Vergangenheitsgrunds ermangelt, der unerlässlich für die Assoziation ist. Was „das Erste der Affektion“, die Uraffektion, betrifft, so ist sie "nicht apperzipierbar als irgendein Seiendes“, auch wenn man versucht, sie aufgrund der Annahme einer besonderen ihr eigenen Seinsweise - wie etwa der Vorgegebenheit - zu erfassen, weil sie selbst nicht in der Zeit, sondern vor der Zeitigung auf der Ebene des Uranfangs liegt. Also besteht die Vor- und Nachbeziehung des Ichs und des Nicht-Ichs auf dieser Ebene nicht. Hierbei handelt es sich um die Unzeit des Ichs des Uranfangs.

Über das Ich des Uranfangs ist schon gesprochen worden: „Ich ist immer schon Ich von Vermögen, Ich von Kinästhesen, aber auch immer schon Ich, das ein Nicht-Ichliches, ein Hyletisches hat". Das Ich des Uranfangs als die Verflechtung des Ichs und des Nicht-Ichlichen ohne Vor- und Nachbeziehung setzt Husserl in den Ursprung der Genesis.

Das Ich des konstitutiven Anfangs ist kein leerer Ich-Pol und der Anfang der Affektion ist nicht völlig unbestimmt, es ist schon Instinkt-Affektion. Das Erste der „Ich“-Intention (Zuwendung) und Erfüllung ist die in der mütterlichen Lebensgeborgenheit, und nicht etwa ein Knall schlechthin etc. Und so ist damit auch die Konstitution der ersten Umwelt von da aus geregelt. ${ }^{26}$

Sowohl „das Ich des konstitutiven Anfangs“ als auch „der Anfang der Affektion" können nicht voneinander getrennt sein und es kann nicht über ihre Vor- und Nachbeziehung gesprochen werden. Beide sind zwar unterscheidbare, aber nicht trennbare "Ich-Affektion“ als Verflechtung des Ichs und des Nicht-Ichlichen. Ist

25 Ibid., S. 472f.

26 Ibid., S. 474f. 
„das Ich des konstitutiven Anfangs“ die in der chaotischen Störung schwebende Leere? Oder ist es „ein Knall schlechthin“, der die ganze Welt und das Ich plötzlich eröffnen würde? Laut Husserl ist dies nicht der Fall. Husserl findet das Ich im Uranfang als Verflechtung der Ich-Affektion ,in der mütterlichen Lebensgeborgenheit“ als wahren Ursprung der Genesis „Urgeburt“ auf. 27 „Das Ego im Uranfang (der Urgeburt) ist schon Ich gerichteter Instinkte" ${ }^{\text {"28. }}$

Schließlich stößt Husserl am Ende seiner Fragestellung nach dem Anfang auf die Verflechtung der Instinkt-Affektion mit dem Konstitutionsanfang und dem Affektionsanfang. Das Ich im Uranfang als Ursprung der Genesis ist die Entstehung des Ich als Individuum und zugleich der Anfang der Beziehung auf das Andere oder das Ich-Fremde. „Das Ego im Uranfang (der Urgeburt)“ ist nichts anderes als das Ich der Instinkte „in der mütterlichen Lebensgeborgenheit“, nämlich (Ur-) Kind im Mutterleib. Das Ich ist mit und innerhalb der Mutter, die letztlich und ursprünglich die Andere ist, aber trotz alledem kann die klare Grenze meiner selbst nicht gezogen werden.

\section{Schluss}

Durch die Auslegung der Frage nach dem Uranfang, die sich im Text Nr. 43 von Lebenswelt (Husserliana Bd. XXXIX) entfaltet, stößt Husserl auf die Verflechtung „Instinkt-Affektion“ von Ich und Nicht-Ichlichem. Aber ist dieser Anfang der Subjektivität als Verflechtung von Instinkt und Affektion wirklich dasjenige, mit dem Husserls Denken einen zufriedenstellenden Abschluss gefunden hat? Nein. Sie ist nicht der letzte Endpunkt der Phänomenologie Husserls, sondern der Ausgangspunkt, der ihr eine neue, nach Lösung verlangende Aufgabe gibt.

Bedeutet die Einführung der Konstruktion als Methode zur Behandlung neuer Probleme wie demjenigen des Uranfangs ein Scheitern oder eine Vertiefung für die Phänomenologie? Wie kann das Ich der Instinkte als „das Ego im Uranfang (der Urgeburt)“ eine Beziehung zum Anderen oder zum Ichfremden und zu seiner Umwelt entwickeln? Diese ungeheuer großen Fragen könnten erst nach der durchgängigen Analyse der ganzen späten Manuskripte Husserls und im Hinblick auf die ganze Konstellation der Phänomenologen, die Husserl und viele andere

27 In diesem Argument Husserls findet Alice Pugliese die Entstehung der „Phänomenologie der Kindheit“. Und sie setzt die Phänomenologie der Kindheit ins Fundament der „Interaktion des Urkindes mit seiner Umgebung, des Einzelsubjekts mit seinem intersubjektiven Netzwerk“. Pugliese A., „Triebsphäre und Urkindheit des Ich“, op. cit., S. 156. 
enthalten, beantwortet werden. Auch in diesem Sinne ist der Uranfang, auf den Husserl in der letzten Phase seines Denkens stößt, zugleich der Uranfang der neuen phänomenologischen Frage.

Takuya Nakamura is Associate Professor in the Department of Philosophy, Faculty of Letters at Doshisha University in Kyoto, Japan. His research interests are transcendental philosophy, in particular Husserl's transcendental phenomenology, phenomenology of time, subjectivity and intersubjectivity, and the ego-problematic in Husserl.

E-mail: taknakam@mail.doshisha.ac.jp 